\title{
Periodicity and Permanence of a Discrete Impulsive Lotka-Volterra Predator-Prey Model Concerning Integrated Pest Management
}

\author{
Chang $\operatorname{Tan}^{1,2}$ and Jun $\mathrm{Cao}^{3}$ \\ ${ }^{1}$ College of Science, Northeast Forestry University, Harbin 150040, China \\ ${ }^{2}$ Forestry Engineering Mobile Station, Northeast Forestry University, Harbin 150040, China \\ ${ }^{3}$ College of Mechanical and Electrical Engineering, Northeast Forestry University, Harbin 150040, China \\ Correspondence should be addressed to Chang Tan; tanchang820@126.com
}

Received 18 September 2013; Revised 23 November 2013; Accepted 25 November 2013

Academic Editor: Stefan Balint

Copyright (C) 2013 C. Tan and J. Cao. This is an open access article distributed under the Creative Commons Attribution License, which permits unrestricted use, distribution, and reproduction in any medium, provided the original work is properly cited.

By piecewise Euler method, a discrete Lotka-Volterra predator-prey model with impulsive effect at fixed moment is proposed and investigated. By using Floquets theorem, we show that a globally asymptotically stable pest-eradication periodic solution exists when the impulsive period is less than some critical value. Further, we prove that the discrete system is permanence if the impulsive period is larger than some critical value. Finally, some numerical experiments are given.

\section{Introduction}

Impulsive equations are found in almost every domain of applied science, such as population dynamics, ecology, biological systems, and optimal control. In recent years, the theory of impulsive differential equations has been an object of active research (see [1-4] and reference therein) since it is much richer than the corresponding theory of differential equations without impulsive effects.

It is well known that continuous-time dynamic systems play an important role in control theory, population dynamics, and so on. But in applications of continuous-time dynamic systems to some practical problems, such as computer simulation, experimental, or computational purposes, it is usual to formulate a discrete-time system which is a version of the continuous-time system. In some sense, the discrete time model inherits the dynamical characteristics of the continuous-time systems. We refer to [4-16] for related discussions of the importance and the need for discretetime analogs to reflect the dynamics of their continuous-time counterparts. Nevertheless, the discrete-time version can but not always preserve the dynamics of its initial version because the theory of difference equations is a lot richer than the corresponding theory of differential equations as pointed out in $[17,18]$. Therefore, it is important to study the dynamics of its initial version alone.

Due to the above facts, we construct the following discrete impulsive Lotka-Volterra predator-prey model concerning integrated pest management by piecewise Euler method:

$$
\begin{gathered}
x_{k T+l+1}=x_{k T+l} \exp \left\{r-a x_{k T+l}-b y_{k T+l}\right\}, \\
y_{k T+l+1}=y_{k T+l} \exp \left\{-d+c x_{k T+l}\right\}, \\
x_{k T+0}=\left(1-p_{1}\right) x_{k T}, \\
y_{k T+0}=\left(1-p_{2}\right) y_{k T}+\mu, \quad l=0,1,2, \ldots, T-1, \\
k=0,1,2, \ldots,
\end{gathered}
$$

where $r>0$ is the intrinsic growth rate of pest, $a>0$ is the coefficient of intraspecific competition, $b>0$ is the per-capita rate of predation of the predator, $d>0$ is the death rate of predator, $c>0$ denotes the product of the per-capita rate of predation and the rate of conversing pest into predator, and $T$ is the period of the impulsive effect. $0 \leq p_{1}<1\left(0 \leq p_{2}<1\right)$ represents the fraction of pest (predator) which dies due to the pesticide, and $\mu>0$ is the release amount of predator at $k T, k \in \mathbb{Z}_{+}$. That is, we can use a combination of biological 
(periodic releasing natural enemies) and chemical (spraying pesticide) tactics that eradicates the pest to extinction and show the efficiency of integrated pest management strategy.

System (1) can be regarded as a discrete analogy of the following impulsive Lotka-Volterra predator-prey model concerning integrated pest management:

$$
\begin{gathered}
\frac{d x_{1}}{d t}=x_{1}\left\{r-a x_{1}-b x_{2}\right\} \\
\frac{d x_{2}}{d t}=x_{2}\left\{-d+c x_{1}\right\} \\
\Delta x_{1}=\left(1-p_{1}\right) x_{1} \\
\Delta x_{2}=\left(1-p_{2}\right) x_{2}+\mu,
\end{gathered}
$$

where $\Delta x_{i}(t)=x_{i}\left(t^{+}\right)-x_{i}(t), i=1,2$. Liu et al. [19] discussed the dynamical behavior of model (2).

Recently, the studies of discrete impulsive model have received great attention from more scholar (see $[5,15,16$, 20-22]). The main difficulty for dynamical analysis of such equations comes from impulsive effect on the equations since the corresponding theory for impulsive difference equations have not yet been fully developed. The discrete impulsive model (1) gives a new form of describing the impulsive moment. In some papers, authors use $(k T)^{+}$to denote impulsive moment (see [20]). It is obvious that describing the impulsive moment of model (1) is easily realized at computer. In addition, some authors use $k T-1$ to denote impulsive moment (see $[16,21])$. Compared with it, model (1) is a better analogue of the continuous-time dynamic system.

The main aim of this paper is to construct the discrete impulsive model (1) and discuss the dynamical behaviors of the discrete impulsive model (1). We investigate the globally asymptotical stability of pest-eradication periodic solution system (1) and the permanence of system (1).

\section{Global Qualitative Analysis for Model (1)}

Before our main results, we will give some lemmas which will be useful for our main results. First, we present the Floquent theory for the linear $T$-periodic difference equation

$$
u_{n+1}=A_{n} u_{n}
$$

where $u_{n} \in R^{s}, n=0,1,2, \ldots A_{n}$ is a real $s \times s$ matrix whose entries are functions of $n$ satisfying $A_{n}=A_{n+T}$ for a positive integer $T$ and is nonsingular; that is, $\operatorname{det} A_{n} \neq 0$ for all $n=0,1,2, \ldots$. As usual $M=A_{T-1} A_{T-2} \cdots A_{0}$ denotes the monodromy of (3).

Lemma 1 (see $[5,6])$. Let $B$ be an $s \times s$ nonsingular matrix and let $T$ be any positive integer. Then there exists some $s \times s$ matrix $C$, such that $C^{T}=B$.

Lemma 2 (see $[5,6]$ ). If we write $M=D^{T}$, then there exists a change of variable $u_{n}=S_{n} y_{n}$, with $S_{n+T}=S_{n}$ for all $n=$ $0,1,2, \ldots$, such that (3) becomes

$$
y_{n+1}=D y_{n}
$$

Lemma 3 (see [5]). Let $a_{k T+l}$ be a solution of the following impulsive inequality:

$$
\begin{gathered}
a_{k T+l+1} \geq a_{k T+l} \exp (-d), \\
a_{k T+0}=\left(1-p_{2}\right) a_{k T}+\mu, \quad l=0,1,2, \ldots, T-1, \\
a_{0+0}=a_{0}, \quad k=0,1,2, \ldots
\end{gathered}
$$

and let $b_{k T+l}$ be a solution of the following impulsive inequality

$$
\begin{gathered}
b_{k T+l+1}=b_{k T+l} \exp (-d), \\
b_{k T+0}=\left(1-p_{2}\right) b_{k T}+\mu, \quad l=0,1,2, \ldots, T-1, \\
b_{0+0}=a_{0}, \quad k=0,1,2, \ldots .
\end{gathered}
$$

Then $a_{k T+l} \geq b_{k T+l}, l=0,1,2, \ldots, T-1$.

Proof. First, we know that $a_{0+0}=a_{0}=b_{0+0}$. By induction, we assume that $a_{k T+l} \geq b_{k T+l}, k=0,1,2, \ldots, l=1,2, \ldots, T-1$.

There are two cases.

Case 1. Considering $l \neq 0, k=0,1,2, \ldots$ or $k=0, l=0,1$, $2, \ldots, T-1$, we have

$$
\begin{aligned}
a_{k T+l+1} & \geq a_{k T+l} \exp (-d) \\
& \geq b_{k T+l} \exp (-d)=b_{k T+l+1} .
\end{aligned}
$$

Case 2. Considering $l=0, k=1,2, \ldots$, we have

$$
\begin{aligned}
a_{k T+1} & \geq a_{k T+0} \exp (-d) \\
& =\left(\left(1-p_{2}\right) a_{k T}+\mu\right) \exp (-d) \\
& \geq\left(\left(1-p_{2}\right) b_{k T}+\mu\right) \exp (-d)=b_{k T+1} .
\end{aligned}
$$

Therefore, for both cases we have $a_{k T+l} \geq b_{k T+l}$. The proof is complete.

Lemma 4. Suppose $\left(x_{k T+l}, y_{k T+l}\right)$ is a solution of (1) subject to $x_{0}>0, y_{0}>0$; then $x_{k T+l}>0, y_{k T+l}>0$ for all $k T+l \geq 0$, $k=0,1,2, \ldots, l=0,1,2, \ldots, T-1$.

2.1. Global Stability of the Pest-Eradication Periodic Solution. Consider the following system:

$$
\begin{gathered}
y_{k T+l+1}=y_{k T+l} \exp (-d), \\
y_{k T+0}=\left(1-p_{2}\right) y_{k T}+\mu, \quad l=0,1,2, \ldots, T-1, \\
y_{0+0}=y_{0}>0, \quad k=0,1,2, \ldots .
\end{gathered}
$$

Clearly, $\widetilde{y_{k T+l}}=\left(\mu /\left(1-\left(1-p_{2}\right) \exp (-d T)\right)\right) \exp (-d l), l=$ $0,1,2, \ldots, T-1$, with $y_{0+0}=\left(\mu /\left(1-\left(1-p_{2}\right) \exp (-d T)\right)\right)$, is a positive periodic solution of (9). Since the solution of (9) is $y_{k T+l}=\left(1-p_{2}\right)^{k}\left(y_{0+0}-\left(\mu /\left(1-\left(1-p_{2}\right) \exp (-d T)\right)\right)\right) \exp (-(k T+$ l)d $)+\widetilde{y_{k T+l}}, l=0,1,2, \ldots, T-1, k=0,1,2,3, \ldots$, we have the following lemma.

Lemma 5. Equation (9) has a positive periodic solution $y_{k T+l}$, $k=0,1,2, \ldots, l=0,1,2, \ldots, T-1$, and, for every solution $y_{k T+l}$ of $(9)$, we have $y_{k T+l} \rightarrow \widetilde{y_{k T+l}}$ as $k \rightarrow \infty$. 

tion:

Therefore, system (1) has a pest-eradication periodic solu-

$$
\left(0, \widetilde{y_{k T+l}}\right)=\left(0, \frac{\mu}{1-\left(1-p_{2}\right) \exp (-d T)} \exp (-d l)\right)
$$

and $y_{0+0}=\mu /\left(1-\left(1-p_{2}\right) \exp (-d T)\right)$.

Now we give the conditions which assure the globally stability of the pest-eradication periodic solution $\left(0, \overline{y_{k T+l}}\right)$.

Theorem 6. Let $\left(x_{k T+l}, y_{k T+l}\right)$ be any solution of (1); then $\left(0, \widehat{y_{k T+l}}\right)$ is globally asymptotically stable provided

$$
\begin{aligned}
r T & -\frac{b \mu(1-\exp (-d T))}{\left(1-\left(1-p_{2}\right) \exp (-d T)\right)(1-\exp (-d))} \\
& <\ln \left(\frac{1}{1-p_{1}}\right) .
\end{aligned}
$$

Proof. Firstly, we proved the local stability. The local stability of periodic solution $\left(0, \widetilde{y_{k T+l}}\right)$ may be determined by considering the behavior of small amplitude perturbations of the solution. Defining $x_{k T+l}=u_{k T+l}, y_{k T+l}=v_{k T+l}+\widetilde{y_{k T+l}}$, there may be written

$$
\left(\begin{array}{c}
u_{k T+l+1} \\
v_{k T+l+1}
\end{array}\right)=\left(\begin{array}{c}
u_{k T+l} \exp \left(r-a u_{k T+l}-b\left(v_{k T+l}+\widetilde{y_{k T+l}}\right)\right) \\
\left(v_{k T+l}+\widetilde{y_{k T+l}}\right) \exp \left(-d+c u_{k T+l}\right)
\end{array}\right) .
$$

Equation (12) can be expanded in a Taylor series: after neglecting higher-order terms, the linearized equations read as

$$
\begin{gathered}
u_{k T+l+1}=\exp \left\{r-b \widetilde{y_{k T+l}}\right\} u_{k T+l}, \\
v_{k T+l+1}=\widetilde{y_{k T+l}} c \exp (-d) u_{k T+l}+\exp (-d) v_{k T+l}, \\
u_{k T+0}=\left(1-p_{1}\right) u_{k T}, \\
v_{k T+0}=\left(1-p_{2}\right) v_{k T}, \quad l=0,1,2, \ldots, T-1, \\
k=0,1,2, \ldots
\end{gathered}
$$

Hence

$$
A_{k T+l}=\left(\begin{array}{cc}
\exp \left(r-b \widetilde{y_{k T+l}}\right) & 0 \\
\widetilde{y_{k T+l}} c \exp (-d) & \exp (-d T)
\end{array}\right)
$$

the fundamental solution matrix is

$$
\begin{aligned}
& M=A_{k T+0} A_{k T+1} A_{k T+2} \cdots A_{k T+T-1}\left(\begin{array}{cc}
1-p_{1} & 0 \\
0 & 1-p_{2}
\end{array}\right) \\
& =\left(\begin{array}{cc}
\exp \left(r T-\frac{b \mu}{1-\left(1-p_{2}\right) \exp (-d T)} \frac{1-\exp (-d T)}{1-\exp (-d)}\right) & 0 \\
* & \exp (-d T)
\end{array}\right)\left(\begin{array}{cc}
1-p_{1} & 0 \\
0 & 1-p_{2}
\end{array}\right) \\
& =\left(\begin{array}{cc}
\left(1-p_{1}\right) \exp \left(r T-\frac{b \mu}{1-\left(1-p_{2}\right) \exp (-d T)} \frac{1-\exp (-d T)}{1-\exp (-d)}\right) & 0 \\
* & \left(1-p_{2}\right) \exp (-d T)
\end{array}\right) .
\end{aligned}
$$

There is no need to calculate the exact form of $(*)$ as it is not required in the analysis that follows.

The stability of the periodic solution $\left(0, \widetilde{y_{k T+l}}\right)$ is determined by the eigenvalues of $M$.

Let $\lambda_{1}, \lambda_{2}$ be eigenvalues of matrix $M$. Then

$$
\begin{aligned}
\lambda_{1}=\left(1-p_{1}\right) & \\
& \times \exp \left(r T-\frac{b \mu}{1-\left(1-p_{2}\right) \exp (-d T)} \frac{1-\exp (-d T)}{1-\exp (-d)}\right), \\
& \lambda_{2}=\left(1-p_{2}\right) \exp (-d T),
\end{aligned}
$$

according to Lemmas 1 and $2,\left(0, \widetilde{y_{k T+l}}\right)$ is locally stable if $\left|\lambda_{1}\right|<1$ and $\left|\lambda_{2}\right|<1$. Obviously, $\lambda_{2}<1$. So, $\left(0, \widetilde{y_{k T+l}}\right)$ is locally stable if $\left|\lambda_{1}\right|<1$. That is,

$$
\begin{aligned}
r T- & \frac{b \mu(1-\exp (-d T))}{\left(1-\left(1-p_{2}\right) \exp (-d T)\right)(1-\exp (-d))} \\
& <\ln \left(\frac{1}{1-p_{1}}\right) .
\end{aligned}
$$

In the following we prove the global attractivity. Choose $\varepsilon>0$ such that

$$
\begin{aligned}
\delta \triangleq & \left(1-p_{1}\right) \\
& \times \exp (r T+b \varepsilon T \\
& \left.\quad-\frac{b \mu(1-\exp (-d T))}{\left(1-\left(1-p_{2}\right) \exp (-d T)\right)(1-\exp (-d))}\right)
\end{aligned}
$$

$<1$. 
Noting that $y_{k T+l+1} \geq y_{k T+l} \exp (-d)$, consider the following impulsive equation:

$$
\begin{gathered}
z_{k T+l+1}=z_{k T+l} \exp \{-d\}, \\
z_{k T+0}=\left(1-p_{2}\right) z_{k T}+\mu, \quad l=0,1,2, \ldots, T-1, \\
k=0,1,2, \ldots, \\
z_{0+0}=y_{0} \geq 0 .
\end{gathered}
$$

By Lemmas 3 and 5, we have

$$
y_{k T+l} \geq z_{k T+l}>\widetilde{y_{k T+l}}-\varepsilon,
$$

for $k$ large enough, so

$$
\begin{aligned}
x_{k T+l+1} & \leq x_{k T+l} \exp \left(r-b y_{k T+l}\right) \\
& \leq x_{k T+l} \exp \left(r-b\left(\widetilde{y_{k T+l}}-\varepsilon\right)\right),
\end{aligned}
$$

which leads to

$$
\begin{aligned}
& x_{k T+0} \\
& =\left(1-p_{1}\right) x_{k T} \\
& \leq\left(1-p_{1}\right) x_{k T-1} \exp \left(r-b\left(\widetilde{y_{k T-1}}-\varepsilon\right)\right) \\
& \leq\left(1-p_{1}\right) x_{k T-2} \exp \left(r-b\left(\widetilde{y_{k T-2}}-\varepsilon\right)\right) \\
& \times \exp \left(r-b\left(\widetilde{y_{k T-1}}-\varepsilon\right)\right) \\
& \leq\left(1-p_{1}\right) x_{(k-1) T+0} \\
& \times \exp (T r+b T \varepsilon \\
& \left.-b\left(\widetilde{y_{(k-1) T+0}}+\widetilde{y_{(k-1) T-1}}+\cdots+\widetilde{y_{k T-1}}\right)\right) \\
& \leq\left(1-p_{1}\right) x_{(k-1) T+0} \\
& \times \exp (\operatorname{Tr}+b T \varepsilon \\
& \left.-\frac{b \mu(1-\exp (-d T))}{\left(1-\left(1-p_{2}\right) \exp (-d T)\right)(1-\exp (-d))}\right) .
\end{aligned}
$$

Therefore, $x_{k T+0} \leq x_{0+0} \delta^{k}$ and $x_{k T+0} \rightarrow 0$ as $k \rightarrow+\infty$. Moreover, $0<x_{k T+l} \leq x_{k T+0} \exp (r T+b T \varepsilon)$, so we have $x_{k T+l} \rightarrow 0$ as $k \rightarrow \infty$.

Next, we prove that $y_{k T+l} \rightarrow \widetilde{y_{k T+l}}$ as $k \rightarrow \infty$ if $\lim _{k \rightarrow \infty} x_{k T+l}=0$. For $0<\varepsilon_{1}<d / c$, there exists an $K_{0}>0$ such that $0<x_{k T+l}<\varepsilon_{1}$ for all $l=0,1,2, \ldots, T-1$, and $k>K_{0}$; then from (1) we have

$$
y_{k T+l} \exp (-d)<y_{k T+l+1}<y_{k T+l} \exp \left(-d+c \varepsilon_{1}\right) .
$$

By Lemmas 3 and 5, we obtain

$$
\begin{gathered}
y_{k T+l}^{1} \leq y_{k T+l} \leq y_{k T+l}^{2}, \\
y_{k T+l}^{1} \longrightarrow \widetilde{y_{k T+l}}, \quad y_{k T+l}^{2} \longrightarrow \widetilde{y_{k T+l}^{2}} \text { as } k \longrightarrow \infty,
\end{gathered}
$$

where $y_{k T+l}^{1}$ and $y_{k T+l}^{2}$ are solutions of

$$
\begin{gathered}
y_{k T+l+1}^{1}=y_{k T+l}^{1} \exp \{-d\}, \\
y_{k T+0}^{1}=\left(1-p_{2}\right) y_{k T}^{1}+\mu, \quad l=0,1,2, \ldots, T-1, \\
k=0,1,2, \ldots, \\
y_{0+0}^{1}=y_{0} \geq 0, \\
y_{k T+l+1}^{2}=y_{k T+l}^{2} \exp \left\{-d+c \varepsilon_{1}\right\}, \\
y_{k T+0}^{2}=\left(1-p_{2}\right) y_{k T}^{2}+\mu, \quad l=0,1,2, \ldots, T-1, \\
y_{0+0}^{2}=y_{0} \geq 0, \quad k=0,1,2, \ldots,
\end{gathered}
$$

respectively, and $\widetilde{y_{k T+l}^{2}}=\left(\mu /\left(1-\left(1-p_{2}\right) \exp ((-d+\right.\right.$ $\left.\left.\left.\left.c \varepsilon_{1}\right) T\right)\right)\right) \exp \left(\left(-d+c \varepsilon_{1}\right) l\right), l=0,1,2, \ldots, T-1$.

Therefore, for any $\varepsilon_{2}>0$ there exists a $\widehat{K}>0$ such that

$$
\widetilde{y_{k T+l}}-\varepsilon_{2}<y_{k T+l}<\widetilde{y_{k T+l}^{2}}+\varepsilon_{2} \text {. }
$$

Let $\varepsilon_{1} \rightarrow 0$; we have

$$
\widetilde{y_{k T+l}^{2}} \longrightarrow \widetilde{y_{k T+l}} \text { as } k \longrightarrow \infty \text {, }
$$

which implies $y_{k T+l} \rightarrow \widetilde{y_{k T+l}}$ as $k \rightarrow \infty$. This completes the proof.

2.2. Permanence. Now we investigate the permanence of system (1). For convenience, let $n=k T+l$. Firstly, we give the following definition.

Definition 7. System (1) is said to be permanent if there are constants $m, M>0$ (independent of initial value) and a finite time $T_{0}$ such that for all solutions $\left(x_{n}, y_{n}\right)$ with all initial values $\left(x_{0+0}>0, y_{0+0}>0\right)$,

$$
m<x_{n}<M, \quad m<y_{n}<M
$$

hold for all $n>T_{0}$. Here $T_{0}$ may depend on the initial values $\left(x_{0+0}, y_{0+0}\right)$.

Lemma 8. There exist two positive constants $M$ and $N$, such that for every solution $\left(x_{n}, y_{n}\right)$ of system (1), we have

$$
x_{n}<M \text { for } n>N \text {. }
$$

Proof. To prove (30), we have two cases.

Case I. $n \neq k T+0, k=0,1,2,3, \ldots$. For any $x_{0}>0$, we have

$$
\begin{aligned}
x_{n+1} & =x_{n} \exp \left\{r-a x_{n}-b y_{n}\right\} \\
& \leq x_{n} \exp \left\{r-a x_{n}\right\} \\
& \leq \frac{\exp \{r-1\}}{a} \triangleq M ;
\end{aligned}
$$


here we used

$$
\max _{x \geq 0}\{x \exp \{b-a x\}\}=\frac{\exp \{b-1\}}{a} .
$$

Case II. $n=k T+0, k=0,1,2,3, \ldots$, we have

$$
\begin{aligned}
x_{k T+0} & =\left(1-p_{1}\right) x_{k T} \\
& =\left(1-p_{1}\right) x_{k T-1} \exp \left\{r-a x_{k T-1}-b y_{k T-1}\right\} \\
& \leq\left(1-p_{1}\right) x_{k T-1} \exp \left\{r-a x_{k T-1}\right\} \\
& \leq\left(1-p_{1}\right) M<M .
\end{aligned}
$$

This completes the proof.

In the following, without loss of generality, we assume $M>d / c$ is large enough.

Lemma 9. There exists a constant $M_{1}$ such that, for every solution $\left(x_{n}, y_{n}\right)$ of (1), we have

$$
\limsup _{n \rightarrow \infty} y_{n}<M_{1} \text {. }
$$

Proof. We first prove that there exists a $K>0$ such that

$$
\liminf _{n \rightarrow \infty} y_{n}<K
$$

There exist $K, \varepsilon_{0}, M_{2}>\mu / p_{2}>0$ such that

$$
\begin{gathered}
\varepsilon_{0}<\frac{d}{c} ; \quad M \exp (r-b K)<\varepsilon_{0} ; \quad K>\frac{\mu}{e^{B}-1+p_{2}}, \\
\frac{\mu}{\left(1-p_{2}\right) \exp (A T)-1}<K+1 \\
\frac{1}{A} \ln \frac{M_{2}}{2(K+1)}>3
\end{gathered}
$$

where $A=-d+c M, B=d-c \varepsilon_{0}$. Now we claim that (35) holds. Otherwise, there would exist $N_{1} \in \mathbb{N}$ and $\varepsilon>0$, such that $y_{n}>K+\varepsilon$ for $n>N_{1}$. By Lemma 8, there exists an $N_{2}>N_{1}$ such that

$$
x_{n}<M, \text { for } n>N_{2} \text {. }
$$

Then if $n>N_{2}$

$$
\begin{aligned}
x_{n+1} & \leq x_{n} \exp (r-b(K+\varepsilon)) \\
& \leq M \exp (r-b K)<\varepsilon_{0} .
\end{aligned}
$$

There are two cases as follows.

$$
\begin{aligned}
& \text { (1) If } n=k T+0>N_{2}+1, \\
& \qquad \begin{aligned}
y_{n+1}=y_{k T+1} \\
\leq y_{k T+0} \exp \left(-d+c x_{k T+0}\right) \\
\leq\left(\left(1-p_{2}\right) y_{k T}+\mu\right) \exp \left(-d+c \varepsilon_{0}\right) \\
\leq\left(\left(1-p_{2}\right) y_{n}+\mu\right) \exp (-B) .
\end{aligned}
\end{aligned}
$$

We claim that $y_{n+1}<y_{n}$. Otherwise, $y_{n+1} \geq y_{n}$, so

$$
\left(\left(1-p_{2}\right) y_{n}+\mu\right) \exp (-B) \geq y_{n} \text {; }
$$

hence

$$
y_{n} \leq \frac{\mu}{e^{B}-\left(1-p_{2}\right)}<K,
$$

which is a contradiction.

(2) If $n \neq k T+0>N_{2}+1$,

$$
\begin{aligned}
y_{n+1} & \leq y_{n} \exp \left(-d+c x_{k T+0}\right) \\
& \leq y_{n} \exp \left(-d+c \varepsilon_{0}\right) \\
& \leq y_{n} \exp (-B)<y_{n} .
\end{aligned}
$$

Therefore, for both cases, we have $y_{n+1}<y_{n}$, so $\lim _{n \rightarrow \infty} y_{n}=0$, which is a contradiction.

For $M_{2}$ given by (37), in the following we will prove that (34) holds.

Otherwise,

$$
\limsup _{n \rightarrow \infty} y_{n}>M_{2} \text {. }
$$

By (35) and (44), there exist

$$
n_{2}>n_{1}>N_{2}
$$

such that

$$
\begin{gathered}
y_{n_{1}} \leq K+1, \quad y_{n_{2}} \geq M_{2}, \\
K+1<y_{n}<M_{2} \quad \text { for } n \in\left(n_{1}, n_{2}\right) .
\end{gathered}
$$

(i) If $n_{1}, n_{2}$ are at the same region $\in[k T,(k+1) T)$, then

$$
\begin{aligned}
y_{n_{2}} & \leq y_{n_{2}-1} \exp (A) \\
& \leq y_{n_{2}-2} \exp (2 A) \\
& \vdots \\
& \leq y_{n_{1}} \exp \left(\left(n_{2}-n_{1}\right) A\right)
\end{aligned}
$$

so

$$
n_{2}-n_{1} \geq \frac{1}{A} \ln \frac{M_{2}}{K+1}>3 .
$$

(ii) If $n_{1}, n_{2}$ are not at the same region, we assume $n_{2} \in$ $[k T,(k+1) T)$ and $n_{1} \in[\widetilde{k} T,(\widetilde{k}+1) T)$. Then

$$
\begin{aligned}
y_{n_{2}} \leq & y_{n_{2}-1} \exp (A) \leq y_{n_{2}-2} \exp (2 A) \\
\vdots & \\
\leq & \left(1-p_{2}\right) y_{k T} \exp \left(\left(n_{2}-k T\right) A\right) \\
& +\mu \exp \left(\left(n_{2}-k T\right) A\right)
\end{aligned}
$$




$$
\begin{aligned}
\leq & \left(1-p_{2}\right)^{2} y_{(k-1) T} \exp \left[\left(n_{2}-(k-1) T\right) A\right] \\
& +\mu\left(1-p_{2}\right) \exp \left[\left(n_{2}-(k-1) T\right) A\right] \\
& +\mu \exp \left(\left(n_{2}-k T\right) A\right) \\
\vdots & \\
\leq & \left(1-p_{2}\right)^{k-\widetilde{k}} y_{(\widetilde{k}+1) T} \exp \left[\left(n_{2}-(\widetilde{k}+1) T\right) A\right] \\
& +\mu\left(1-p_{2}\right)^{k-\tilde{k}-1} \exp \left[\left(n_{2}-(\widetilde{k}+1) T\right) A\right] \\
& +\mu\left(1-p_{2}\right)^{k-\widetilde{k}-2} \exp \left[\left(n_{2}-(\widetilde{k}+2)\right) T A\right] \\
& +\cdots+\mu\left(1-p_{2}\right)^{2} \exp \left[\left(n_{2}-(k-2) T\right) A\right] \\
& +\mu\left(1-p_{2}\right) \exp \left[\left(n_{2}-(k-1) T\right) A\right] \\
& +\mu \exp \left(\left(n_{2}-k T\right) A\right) \\
\leq & \left(1-p_{2}\right)^{k-\widetilde{k}} y_{n_{1}} \exp \left(\left(n_{2}-n_{1}\right) A\right) \\
& +\mu \exp \left(\left(n_{2}-k T\right) A\right)\left[1-\left(1-p_{2}\right) \exp [(k-\widetilde{k}) A T]\right] \\
\leq & y_{n_{1}} \exp \left(\left(n_{2}-n_{1}\right) A\right) \\
& +\frac{\mu\left(1-p_{2}\right) \exp \left[\left(n_{2}-\widetilde{k} T\right) A-\exp \left(\left(n_{2}-k T\right) A\right)\right]}{\left(1-p_{2}\right) \exp (A T)-1} \\
\leq & 2(K+1) \exp \left[\left(n_{2}-n_{1}\right) A\right] . \\
& \left.\frac{\left.p_{2}\right) \exp (A T)}{1-1}\right)
\end{aligned}
$$

So

$$
n_{2}-n_{1} \geq \frac{1}{A} \ln \frac{M_{2}}{2(K+1)}>3 .
$$

Therefore, the set $\left\{n \mid n_{1}<n<n_{2}\right\}$ is nonempty. So if $n_{2}-1 \neq k T+0>N_{2}+1$, then

$$
\begin{aligned}
x_{n_{2}-1} & =x_{n_{2}-2} \exp \left(r-a x_{n_{2}-2}-b y_{n_{2}-2}\right) \\
& \leq x_{n_{2}-2} \exp \left(r-b y_{n_{2}-2}\right) \\
& \leq M \exp (r-b(K+1)) \\
& \leq \varepsilon_{0} .
\end{aligned}
$$

If $n_{2}-1=k T+0>N_{2}+1$, then

$$
\begin{aligned}
x_{n_{2}-1} & =x_{k T+0}=\left(1-p_{1}\right) x_{k T} \\
& \leq\left(1-p_{1}\right) x_{k T-1} \exp (r-b(K+1)) \\
& \leq M \exp (r-b(K+1)) \\
& \leq \varepsilon_{0} .
\end{aligned}
$$

There are three case as follows.
(1) If $n_{2}=k T+1, k=1,2,3, \ldots$ and $M_{2}>\mu / p_{2}$,

$$
\begin{aligned}
y_{n_{2}} & =y_{k T+1} \\
& =y_{k T+0} \exp \left(-d+c x_{k T+0}\right) \\
& =\left(\left(1-p_{2}\right) y_{k T}+\mu\right) \exp \left(-d+c x_{k T+0}\right) \\
& \leq\left(\left(1-p_{2}\right) y_{k T}+\mu\right) \exp \left(-d+c \varepsilon_{0}\right) \\
& \leq\left(\left(1-p_{2}\right) M_{2}+\mu\right) \exp \left(-d+c \varepsilon_{0}\right) \\
& \leq\left(1-p_{2}\right) M_{2}+\mu \leq M_{2} .
\end{aligned}
$$

(2) If $n_{2}=k T+0, k=1,2,3, \ldots$ and $M_{2}>\mu / p_{2}$,

$$
\begin{aligned}
y_{n_{2}} & =y_{k T+0} \\
& =\left(\left(1-p_{2}\right) y_{k T}+\mu\right) \\
& =\left(\left(1-p_{2}\right) y_{k T-1} \exp \left(-d+c x_{k T-1}\right)+\mu\right) \\
& \leq\left(\left(1-p_{2}\right) y_{k T-1} \exp \left(-d+c \varepsilon_{0}\right)+\mu\right) \\
& \leq\left(\left(1-p_{2}\right) M_{2} \exp \left(-d+c \varepsilon_{0}\right)+\mu\right) \leq M_{2} .
\end{aligned}
$$

(3) Other cases are as follows:

$$
\begin{aligned}
y_{n_{2}} & =y_{n_{2}-1} \exp \left(-d+c x_{n_{2}-1}\right) \\
& =y_{n_{2}-1} \exp \left(-d+c x_{n_{2}-1}\right) \\
& \leq M_{2} \exp \left(-d+c \varepsilon_{0}\right) \leq M_{2},
\end{aligned}
$$

which is a contradiction. This completes the proof.

Theorem 10. Equation (1) is permanence provided that

$$
\begin{aligned}
r T & -\frac{b \mu(1-\exp (-d T))}{\left(1-\left(1-p_{2}\right) \exp (-d T)\right)(1-\exp (-d))} \\
& >\ln \left(\frac{1}{1-p_{1}}\right)
\end{aligned}
$$

holds true.

Proof. Suppose $\left(x_{n}, y_{n}\right)$ is a solution of (1) with $\left(x_{0}>0, y_{0}>\right.$ 0 ). By Lemmas 8 and 9 , we have proved there exists a constant $M>0$ such that $\left(x_{n}<M, y_{n}<M\right)$ for $n$ large enough.

From (20), we know $y_{n}>\tilde{y}_{n}-\varepsilon$ for all $n$ large enough and some $\varepsilon>0$, so $y_{n} \geq\left(\mu /\left(1-\left(1-p_{2}\right) \exp (-d T)\right)\right) \exp (-d T)-\varepsilon \triangleq$ $m_{2}$ for $n$ large enough.

Thus, we only need to find $m_{1}>0$ such that $x_{n} \geq m_{1}$ for $n$ large enough.

We will do it in the following two steps. 
Step I. From condition (56), let $0<m_{3}<d / c, \varepsilon_{1}>0$ be small enough such that $\eta \triangleq\left(1-p_{1}\right) \exp \left(T\left(r-a m_{3}-b \varepsilon_{1}\right)-(\mu /(1-\right.$ $\left.\left.\left(1-p_{2}\right) \exp \left(\left(-d+c m_{3}\right) T\right)\right)\right)\left(b\left(1-\exp \left(\left(-d+c m_{3}\right) T\right)\right) /(1-\right.$ $\left.\left.\left.\exp \left(-d+c m_{3}\right)\right)\right)\right)>1$; we will prove that $x_{n}<m_{3}$ cannot hold for all $n>0$. Otherwise,

$$
\begin{gathered}
y_{n+1} \leq y_{n} \exp \left(-d+c m_{3}\right), \\
y_{k T+0}=\left(1-p_{2}\right) y_{k T}+\mu .
\end{gathered}
$$

So we have $y_{n} \leq z_{n}$ and $z_{n} \rightarrow \widetilde{z}_{n}, n \rightarrow \infty$, where $z_{n}$ is the solution of

$$
\begin{gathered}
z_{n+1}=z_{n} \exp \left(-d+c m_{3}\right), \\
z_{k T+0}=\left(1-p_{2}\right) z_{k T}+\mu, \\
z_{0+0}=y_{0+0},
\end{gathered}
$$

and $\widetilde{z}_{n}=\left(\mu /\left(1-\left(1-p_{2}\right) \exp \left(\left(-d+c m_{3}\right) T\right)\right)\right) \exp ((-d+$ $\left.\left.c m_{3}\right) l\right), l=0,1,2, \ldots, T-1$, with $y_{0+0}=\mu /(1-(1-$ $\left.\left.p_{2}\right) \exp \left(\left(-d+c m_{3}\right) T\right)\right)$, is a positive periodic solution of (58).

Therefore, there exists a $N_{1}>0$ such that

$$
\begin{gathered}
y_{n} \leq z_{n}<\widetilde{z}_{n}+\varepsilon_{1}, \\
x_{n+1} \geq x_{n} \exp \left(r-a m_{3}-b\left(\widetilde{z}_{n}+\varepsilon_{1}\right)\right), \\
x_{k T+0}=\left(1-p_{1}\right) x_{k T}
\end{gathered}
$$

for $n>N_{1}$. Therefore there exists a $K_{1}$, such that if $k>K_{1}$

$$
\begin{aligned}
& x_{k T+T} \geq x_{k T+T-1} \exp \left(r-a m_{3}-b\left(\widetilde{z_{k T+T-1}}+\varepsilon_{1}\right)\right) \\
& \geq x_{k T+T-1} \exp \left(r-a m_{3}-b \varepsilon_{1}\right) \exp \left(-\widetilde{b z_{k T+T-1}}\right) \\
& \geq x_{k T+T-2} \exp \left(2\left(r-a m_{3}-b \varepsilon_{1}\right)\right) \\
& \times \exp \left(-b\left(\widetilde{z_{k T+T-1}}+\widetilde{z_{k T+T-2}}\right)\right) \\
& \geq: \\
& \geq x_{k T+0} \exp \left(T\left(r-a m_{3}-b \varepsilon_{1}\right)\right) \\
& \times \exp \left(-b\left(\widetilde{z_{k T+T-1}}+\widetilde{z_{k T+T-2}}+\cdots+\widetilde{z_{k T+0)}}\right)\right) \\
& \geq\left(1-p_{1}\right) x_{k T} \\
& \times \exp \left(T\left(r-a m_{3}-b \varepsilon_{1}\right)\right. \\
& -\frac{\mu}{1-\left(1-p_{2}\right) \exp \left(\left(-d+c m_{3}\right) T\right)} \\
& \left.\times \frac{b\left(1-\exp \left(\left(-d+c m_{3}\right) T\right)\right)}{1-\exp \left(-d+c m_{3}\right)}\right) \\
& =x_{k T} \eta
\end{aligned}
$$

then $x_{\left(k+K_{1}\right) T} \geq x_{K_{1} T} \eta^{k} \rightarrow \infty$ as $k \rightarrow \infty$, which is a contradiction to the boundedness of $x_{k T+l}$. Hence there exists a $n_{1}>0$ such that $x_{n_{1}} \geq m_{3}$.

Step II. If $x_{n} \geq m_{3}$ for all $n \geq n_{1}$, then our aim is achieved. Otherwise, $x_{n}<m_{3}$ for some $n>n_{1}$. Set $n^{*}=\min _{n>n_{1}}\{n \mid$ $\left.x_{n}<m_{3}\right\}$. We have $x_{n} \geq m_{3}$ for $n \in\left[n_{1}, n^{*}\right)$.

Assume that $n^{*} \in[k T,(k+1) T)$. It is easy to see that $x_{n^{*}}<$ $m_{3}, x_{n^{*}-1} \geq m_{3}$.

There are two possible cases for $n \in\left[n^{*},(k+1) T\right)$. Here, for $M$ large enough, we have $\eta_{1}=r-a m_{3}-b M<0$ and $\eta_{2}=r-a M-b M<0$.

Case (a). There exists a $n^{\prime \prime} \in\left[n^{*},(k+1) T\right)$, such that $x_{n^{\prime \prime}} \geq m_{3}$. Let $\widehat{n}=\min _{n>n^{*}}\left\{n \mid x_{n} \geq m_{3}\right\}$; then $x_{n}<m_{3}$ for $n \in\left[n^{*}, \widehat{n}\right)$ and $x_{\widehat{n}} \geq m_{3}$.

For $n \in\left[n^{*}, \widehat{n}-1\right)$,

$$
\begin{aligned}
x_{n+1} & \geq x_{n} \exp \left(\eta_{1}\right) \\
& \vdots \\
& \geq x_{n^{*}} \exp \left(\left(n+1-n^{*}\right) \eta_{1}\right) .
\end{aligned}
$$

$$
\begin{aligned}
& \text { If } n^{* \neq k T+} 0 \text {, then } \\
& \qquad \begin{aligned}
x_{n^{*}} & =x_{n^{*}-1} \exp \left(r-a x_{n^{*}-1}-b y_{n^{*}-1}\right) \\
& \geq m_{3} \exp \left(\eta_{2}\right) \\
x_{n+1} & \geq x_{n^{*}} \exp \left(\left(n+1-n^{*}\right) \eta_{1}\right) \\
& \geq m_{3} \exp \left(\eta_{2}\right) \exp \left(\eta_{1}\right) \\
& \geq\left(1-p_{1}\right) m_{3} \exp \left(\eta_{2}\right) \exp \left(\eta_{1}\right) .
\end{aligned}
\end{aligned}
$$

If $n^{*}=k T+0$, then $k T+0>n_{1}$.

Case (1). Consider $k T=n_{1}$,

$$
x_{n^{*}}=\left(1-p_{1}\right) x_{k T} \geq\left(1-p_{1}\right) m_{3} \geq\left(1-p_{1}\right) m_{3} \exp \left(\eta_{2}\right) \text {. }
$$

Case (2). Consider $k T>n_{1}$,

$$
\begin{aligned}
x_{n^{*}} & =\left(1-p_{1}\right) x_{k T} \\
& \geq\left(1-p_{1}\right) x_{k T-1} \exp \left(r-a x_{k T-1}-b y_{k T-1}\right) \\
& \geq\left(1-p_{1}\right) m_{3} \exp \left(\eta_{2}\right), \\
x_{n+1} & \geq x_{n^{*}} \exp \left(\left(n+1-n^{*}\right) \eta_{1}\right) \\
& \geq\left(1-p_{1}\right) m_{3} \exp \left(\eta_{2}\right) \exp \left(\eta_{1}\right) \\
& \geq\left(1-p_{1}\right) m_{3} \exp \left(\eta_{2}\right) \exp \left(\eta_{1}\right) .
\end{aligned}
$$

Let $m_{1}=\left(1-p_{1}\right) m_{3} \exp \left(\eta_{2}\right) \exp \left(\eta_{1}\right)$. So we have $x_{n} \geq m_{1}$ for $n \in\left[n^{*}, \widehat{n}-1\right)$. For $n \geq \widehat{n}$ the same arguments can be continued since $x_{\widehat{n}} \geq m_{3}$.

Case (b). There does not exist a $n^{\prime \prime} \in\left[n^{*},(k+1) T\right)$, such that $x_{n^{\prime \prime}} \geq m_{3}$; namely, $x_{n}<m_{3}$ for $n \in\left[n^{*},(k+1) T\right)$. 
Choose $n_{2}, n_{3} \in \mathbb{Z}^{+}$such that

$$
\begin{gathered}
n_{2} T>\frac{1}{-d+c m_{3}} \ln \frac{\varepsilon_{1}^{\prime}}{M+\mu}, \\
\exp \left(\eta_{2}\right)\left(1-p_{1}\right)^{n_{2}+1} \exp \left(\eta_{1}\left(n_{2}+1\right) T\right) \eta_{1}^{n_{3}}>1 .
\end{gathered}
$$

Let $T^{\prime}=\left(n_{2}+n_{3}\right) T$. We claim that there must be a $\tilde{n} \in$ $\left[(k+1) T,(k+1) T+T^{\prime}\right)$, such that $x_{\tilde{n}} \geq m_{3}$. Otherwise, $x_{n}<$ $m_{3}, n \in\left[(k+1) T,(k+1) T+T^{\prime}\right)$. Consider (58) with $z_{(k+1) T+0}=$ $y_{(k+1) T+0}$; we have

$$
\begin{aligned}
z_{m T+l}= & \left(z_{(k+1) T+0}-\frac{\mu}{1-\left(1-p_{1}\right) \exp \left(\left(-d+c m_{3}\right) T\right)}\right) \\
& \times \exp \left(\left(-d+c m_{3}\right)(m T+l-(k+1) T)\right)+\widetilde{z}_{m T+l}
\end{aligned}
$$

for $m T+l \in(n T,(n+1) T)$ and $k+1 \leq n \leq k+1+n_{2}+n_{3}$. Then for $m \in\left(k+1+n_{2}, k+1+n_{2}+n_{3}\right)$,

$$
\begin{gathered}
\left|z_{m T+l}-\widetilde{z}_{m T+l}\right| \\
<(M+\mu) \exp \left(\left(-d+c m_{3}\right)(m T+l-(k+1) T)\right) \\
\leq(M+\mu) \exp \left(\left(-d+c m_{3}\right)(m T-(k+1) T)\right) \\
\leq(M+\mu) \exp \left(\left(-d+c m_{3}\right)\left(n_{2} T\right)\right)<\varepsilon_{1}^{\prime}, \\
y_{m T+l} \leq z_{m T+l} \leq \widetilde{z}_{m T+l}+\varepsilon_{1}^{\prime},
\end{gathered}
$$

which implies that (60) holds for $m \in\left(k+1+n_{2}, k+1+n_{2}+n_{3}\right)$.

So as in Step I, we have

$$
x_{\left(k+1+n_{2}+n_{3}\right) T} \geq x_{\left(k+1+n_{2}\right) T} \eta^{n_{3}} .
$$

Since $x_{n}<m_{3}, n \in\left[n^{*},(k+1) T\right)$, then $x_{n}<m_{3}$ for all $n \in\left[n^{*},\left((k+1)+n_{2}\right) T\right]$.

So,

$$
x_{n+1} \geq x_{n} \exp \left(\eta_{1}\right)
$$

$$
\begin{aligned}
x_{\left((k+1)+n_{2}\right) T} & \geq x_{\left((k+1)+n_{2}\right) T-1} \exp \left(\eta_{1}\right) \\
& \geq \vdots \\
& \geq x_{n^{*}}\left(1-p_{1}\right)^{n_{2}} \exp \left(\eta_{1}\left(\left(k+1+n_{2}\right) T\right)-n^{*}\right) .
\end{aligned}
$$

If $n^{*} \neq k T+0$, then

$$
\begin{aligned}
x_{\left((k+1)+n_{2}\right) T} \\
=x_{n^{*}-1} \exp \left[r-a x_{n^{*}-1}-b y_{n^{*}-1}\right] \\
\quad \times\left(1-p_{1}\right)^{n_{2}} \exp \left(\eta_{1}\left(\left(k+1+n_{2}\right) T\right)-n^{*}\right) \\
\geq m_{3} \exp \left(\eta_{2}\right)\left(1-p_{1}\right)^{n_{2}} \exp \left(\eta_{1}\left(\left(k+1+n_{2}\right) T\right)-n^{*}\right) \\
\geq m_{3} \exp \left(\eta_{2}\right)\left(1-p_{1}\right)^{n_{2}} \exp \left(\eta_{1}\left(n_{2}+1\right) T\right) .
\end{aligned}
$$

$$
\text { If } n^{*}=k T+0 \text {, then } k T+0>n_{1}
$$

$x_{\left((k+1)+n_{2}\right) T}$

$$
\begin{aligned}
= & x_{n^{*}}\left(1-p_{1}\right)^{n_{2}+1} \exp \left(\eta_{1}\left(\left(k+1+n_{2}\right) T\right)-n^{*}\right) \\
= & x_{n^{*}-1} \exp \left[r-a x_{n^{*}-1}-b y_{n^{*}-1}\right] \\
& \times\left(1-p_{1}\right)^{n_{2}+1} \exp \left(\eta_{1}\left(\left(k+1+n_{2}\right) T\right)-n^{*}\right) \\
\geq & m_{3} \exp \left(\eta_{2}\right)\left(1-p_{1}\right)^{n_{2}+1} \exp \left(\eta_{1}\left(\left(k+1+n_{2}\right) T\right)-n^{*}\right) \\
\geq & m_{3} \exp \left(\eta_{2}\right)\left(1-p_{1}\right)^{n_{2}+1} \exp \left(\eta_{1}\left(n_{2}+1\right) T\right) .
\end{aligned}
$$

So,

$$
\begin{aligned}
x_{(k+1)+n_{2}+n_{3}} \geq & m_{3} \exp \left(\eta_{2}\right)\left(1-p_{1}\right)^{n_{2}+1} \\
& \times \exp \left(\eta_{1}\left(n_{2}+1\right) T\right) \eta_{1}^{n_{3}}>m_{3},
\end{aligned}
$$

which is a contradiction.

Let $\bar{n}=\min _{n>n^{*}}\left\{n \mid x_{n} \geq m_{3}\right\}$. Then $x_{\bar{n}} \geq m_{3}, x_{\bar{n}-1}<m_{3}$. For $n \in\left[n^{*}, \bar{n}-1\right],(70)$ holds. Suppose $\bar{n} \in\left[(k+1) T+\left(k^{\prime}-\right.\right.$ $\left.\left.1) T,(k+1) T+k^{\prime} T\right)\right], k^{\prime} \leq n_{2}+n_{3}$.

We have

$$
x_{n} \geq x_{n_{1}} \exp \left(\eta_{1}\right)
$$

$$
\geq x_{n^{*}}\left(1-p_{1}\right)^{k^{\prime}} \exp \left(\left(n-n^{*}\right) \eta_{1}\right) \text {. }
$$

If $n^{*} \neq k T+0$, then

$$
\begin{aligned}
& x_{n} \geq m_{3} \exp \left(\eta_{2}\right)\left(1-p_{1}\right)^{k^{\prime}} \exp \left(\left(n-n^{*}\right) \eta_{1}\right) \\
& \quad \geq m_{3} \exp \left(\eta_{2}\right)\left(1-p_{1}\right)^{k^{\prime}} \exp \left(\left(n_{2}+n_{3}+1\right) T \eta_{1}\right) . \\
& \text { If } n^{*}=k T+0 \text {, then } k T+0>n_{1} \\
& x_{n} \geq m_{3} \exp \left(\eta_{2}\right)\left(1-p_{1}\right)^{k^{\prime}+1} \exp \left(\left(n-n^{*}\right) \eta_{1}\right) \\
& \geq m_{3} \exp \left(\eta_{2}\right)\left(1-p_{1}\right)^{k^{\prime}+1} \exp \left(\left(n_{2}+n_{3}+1\right) T \eta_{1}\right) .
\end{aligned}
$$

Let $m_{1}^{\prime}=m_{3} \exp \left(\eta_{2}\right)\left(1-p_{1}\right)^{n_{2}+n_{3}+1} \exp \left(\left(n_{2}+n_{3}+1\right) \eta_{1} T\right)<m_{1}$. So $x_{n}>m_{1}^{\prime}$ for $\left[n^{*}, \bar{n}-1\right]$. For $n>\bar{n}-1$, the same arguments can be continued since $x_{\bar{n}} \geq m_{3}$.

The proof is completed.

Remark 11. Let

$$
\begin{aligned}
f(T)= & r T-\frac{b \mu(1-\exp (-d T))}{\left(1-\left(1-p_{2}\right) \exp (-d T)\right)(1-\exp (-d))} \\
& -\ln \left(\frac{1}{1-p_{1}}\right) .
\end{aligned}
$$




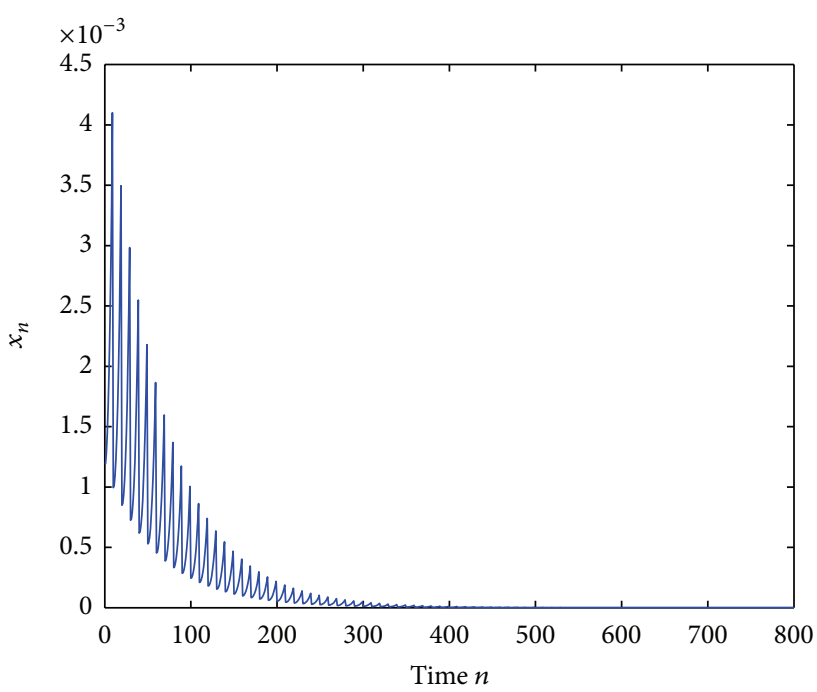

(a) Time-series of the pest population

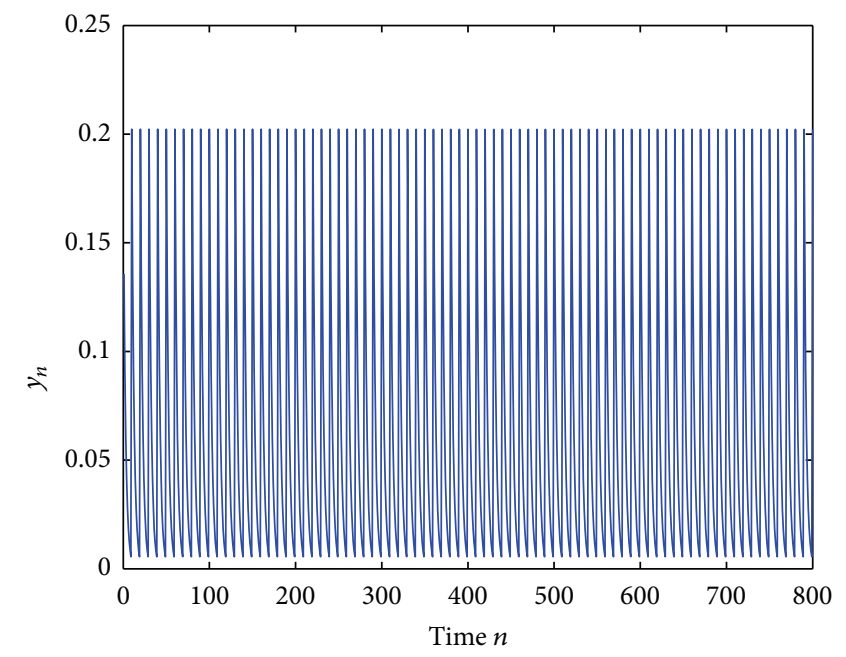

(b) Time-series of the predator population

FIGURE 1: Dynamical behavior of the pest-predator system of (1).

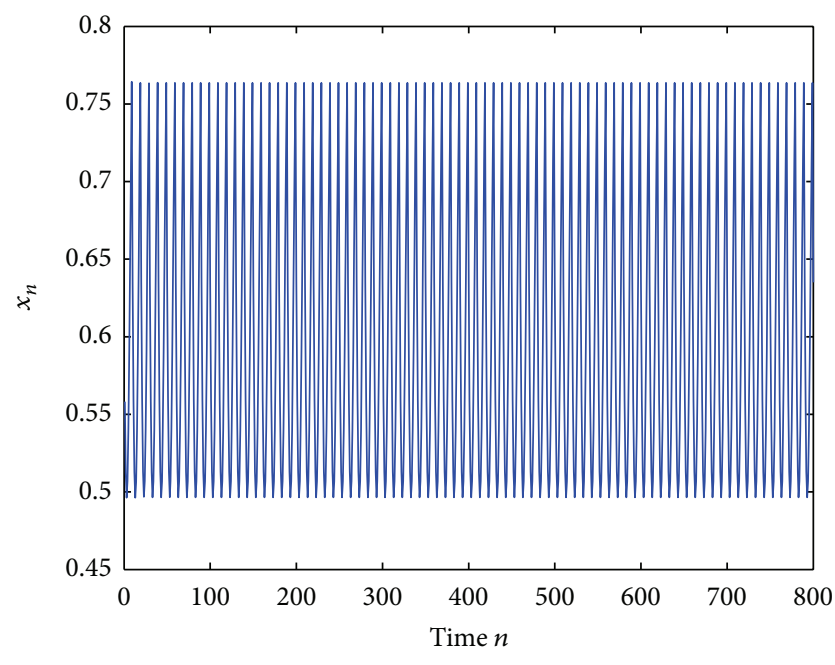

(a) Time-series of the pest population

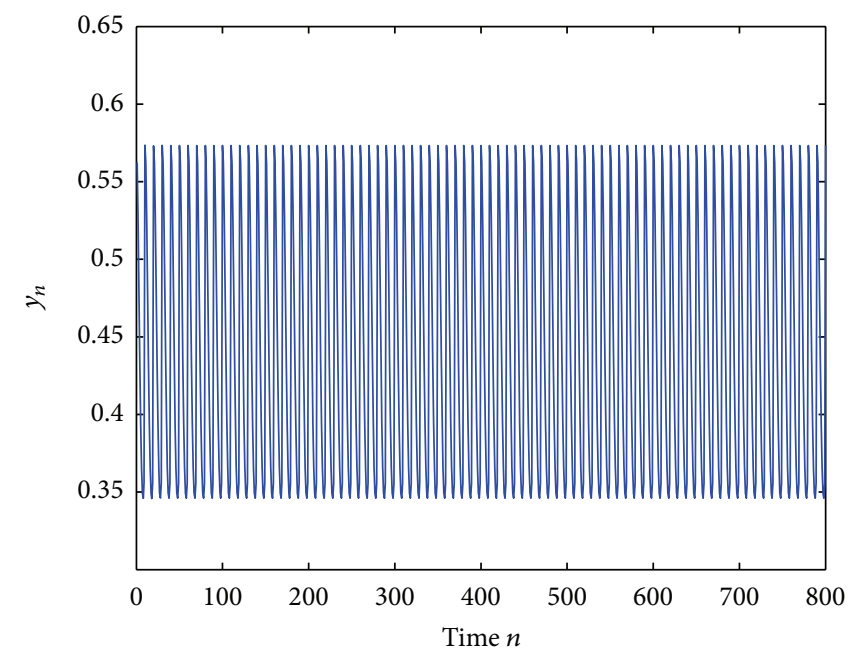

(b) Time-series of the predator population

FIgURE 2: Dynamical behavior of the pest-predator system of (1).

Since $f(0)=-\ln \left(1 /\left(1-p_{1}\right)\right)<0, f(T) \rightarrow \infty$ as $T \rightarrow \infty$ and

$$
\begin{aligned}
f^{\prime \prime}(T) & =\frac{b \mu p_{2} d^{2}}{1-\exp (-d)} \frac{\exp (-d T)\left(1+\left(1-p_{2}\right) \exp (-d T)\right)}{\left(1-\left(1-p_{2}\right) \exp (-d T)\right)^{3}} \\
& >0
\end{aligned}
$$

so $f(T)=0$ has a unique positive root, denoted by $T_{\max }$. From Theorem 6 we know that the pest-eradication periodic solution is globally stable when $T<T_{\max }$. From Theorem 10, system (1) is permanent if $T>T_{\max }$.

\section{Numerical Experiments}

In Figure 1, we choose parameters of system (1) as $d=0.4$, $a=0.4, b=0.9, c=0.6, r=0.2, p_{1}=0.8, p_{2}=0.4, \mu=0.2$, $T=10$, and initial value $x_{0}=1, y_{0}=1$. It is easy to verify that condition (11) holds, and, by Theorem 6 , the pest-eradication periodic solution of (1) is the global stability.

In Figure 2, we choose parameters of system (1) as $d=0.4$, $a=0.4, b=1, c=0.6, r=0.3, p_{1}=0.2, p_{2}=0.01, \mu=0.2$, $T=10$, and initial value $x_{0}=1, y_{0}=1$. It is easy to verify that condition (56) holds, and, by Theorem 10, (1) is permanence.

\section{Conclusion}

In this paper, by piecewise Euler method, we construct a discrete impulsive Lotka-Volterra predator-prey model 
concerning integrated pest management. The discrete impulsive model gives a new form of describing the impulsive moment. On the other hand, model (1) is a better analogue of the continuous-time impulsive dynamic system. By using Floquets theorem, we show that a globally asymptotically stable pest-eradication periodic solution exists when the impulsive period is less than some critical value and the discrete system is permanence if the impulsive period is larger than some critical value. By (78), the impulsive period critical value $T_{\max }$ can be obtained. Since $T_{\max }$ is a direct function with respect to $p_{1}, p_{2}$, and $\mu$, in order to obtain the object of integrated pest management, we can determine the impulsive period $T$ according to effect of the chemical pesticides on the populations and cost of the releasing natural enemies.

\section{Conflict of Interests}

The authors declare that there is no conflict of interests regarding the publication of this paper.

\section{Acknowledgments}

This work is supported by the Fundamental Research Funds for the Central Universities (DL12BB23) and the China Postdoctoral Science Foundation (415220).

\section{References}

[1] V. Lakshmikantham, D. D. Baĭnov, and P. S. Simeonov, Theory of Impulsive Differential Equations, vol. 6, World Scientific Publishing, Teaneck, NJ, USA, 1989.

[2] V. Lakshmikantham, X. Liu, and S. Sathananthan, "Impulsive integro-differential equations and extension of Lyapunov's method," Applicable Analysis, vol. 32, no. 3-4, pp. 203-214, 1989.

[3] D. Baĭnov and P. Simeonov, Impulsive Differential Equations: Periodic Solutions and Applications, vol. 66 of Pitman Monographs and Surveys in Pure and Applied Mathematics, Longman, Harlow, UK, 1993.

[4] K. Gopalsamy and B. G. Zhang, "On delay differential equations with impulses," Journal of Mathematical Analysis and Applications, vol. 139, no. 1, pp. 110-122, 1989.

[5] H. Liang, M. Liu, and M. Song, "Extinction and permanence of the numerical solution of a two-prey one-predator system with impulsive effect," International Journal of Computer Mathematics, vol. 88, no. 6, pp. 1305-1325, 2011.

[6] S. Elaydi, An Introduction to Difference Equations, Springer, New York, NY, USA, 3rd edition, 2005.

[7] J. M. Cushing and S. M. Henson, "Global dynamics of some periodically forced, monotone difference equations," Journal of Difference Equations and Applications, vol. 7, no. 6, pp. 859-872, 2001.

[8] S. Mohamad, "Global exponential stability in discrete-time analogues of delayed cellular neural networks," Journal of Difference Equations and Applications, vol. 9, no. 6, pp. 559-575, 2003.

[9] S. Mohamad and A. G. Naim, "Discrete-time analogues of integrodifferential equations modelling bidirectional neural networks," Journal of Computational and Applied Mathematics, vol. 138, no. 1, pp. 1-20, 2002.
[10] K. Murakami, "Stability for non-hyperbolic fixed points of scalar difference equations," Journal of Mathematical Analysis and Applications, vol. 310, no. 2, pp. 492-505, 2005.

[11] A. M. Stuart and A. R. Humphries, Dynamical Systems and Numerical Analysis, vol. 2, Cambridge University Press, Cambridge, UK, 1996.

[12] Q. Zhang, "On a linear delay difference equation with impulses," Annals of Differential Equations, vol. 18, no. 2, pp. 197-204, 2002.

[13] Z. He and X. Zhang, "Monotone iterative technique for first order impulsive difference equations with periodic boundary conditions," Applied Mathematics and Computation, vol. 156, no. 3, pp. 605-620, 2004.

[14] R. Z. Abdullin, "Stability of nonlinear difference equations with pulse actions: a comparison method," Automation and Remote Control 1, vol. 61, no. 11, pp. 1796-1807, 2000.

[15] R. Z. Abdullin, "Stability of difference equations with impulsive actions at the instants of time dependent on the state vector," Automation and Remote Control 1, vol. 58, no. 7, pp. 1092-1100, 1997.

[16] B. Liu and D. J. Hill, "Uniform stability and ISS of discrete-time impulsive hybrid systems," Nonlinear Analysis: Hybrid Systems, vol. 4, no. 2, pp. 319-333, 2010.

[17] S. Mohamad and K. Gopalsamy, "Exponential stability of continuous-time and discrete-time cellular neural networks with delays," Applied Mathematics and Computation, vol. 135, no. 1, pp. 17-38, 2003.

[18] S. Mohamad and K. Gopalsamy, "Dynamics of a class of discrete-time neural networks and their continuous-time counterparts," Mathematics and Computers in Simulation, vol. 53, no. 1-2, pp. 1-39, 2000.

[19] B. Liu, Y. Zhang, and L. Chen, “The dynamical behaviors of a Lotka-Volterra predator-prey model concerning integrated pest management," Nonlinear Analysis: Real World Applications, vol. 6, no. 2, pp. 227-243, 2005.

[20] Z. Zhang and X. Liu, "Robust stability of uncertain discrete impulsive switching systems," Computers \& Mathematics with Applications, vol. 58, no. 2, pp. 380-389, 2009.

[21] S. Wu, C. Li, X. Liao, and S. Duan, "Exponential stability of impulsive discrete systems with time delay and applications in stochastic neural networks: a Razumikhin approach," Neurocomputing, vol. 82, pp. 29-36, 2012.

[22] Y. Zhang, "Exponential stability of impulsive discrete systems with time delays," Applied Mathematics Letters of Rapid Publication, vol. 25, no. 12, pp. 2290-2297, 2012. 


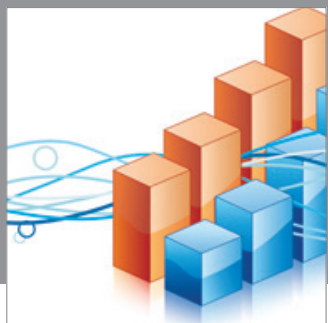

Advances in

Operations Research

mansans

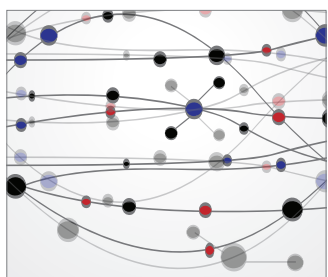

The Scientific World Journal
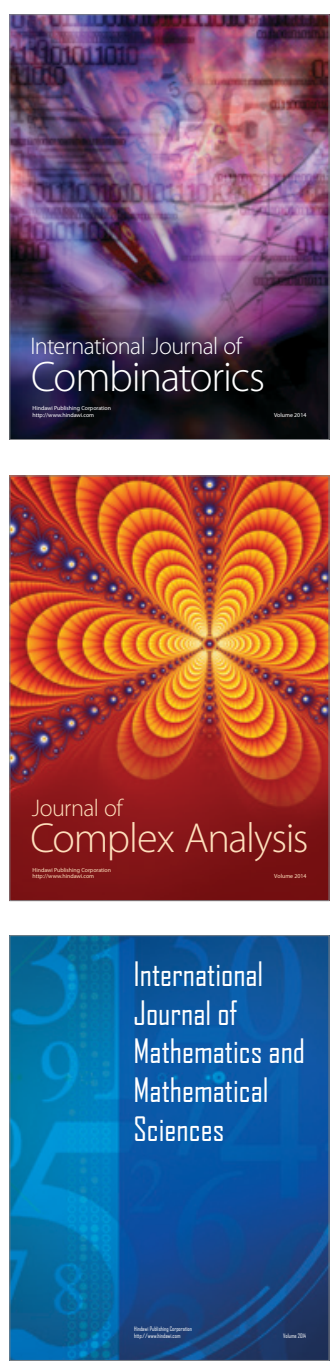
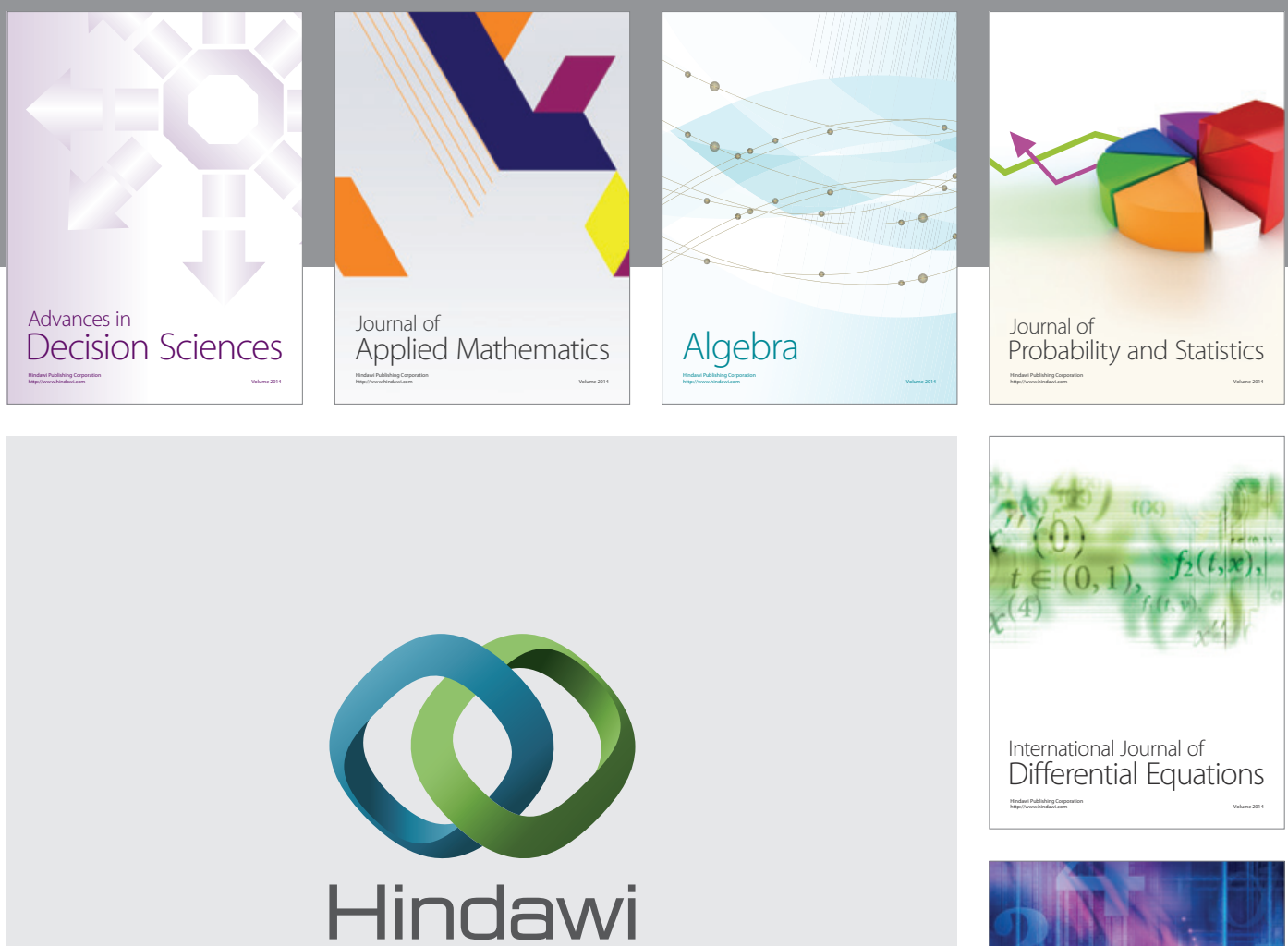

Submit your manuscripts at http://www.hindawi.com
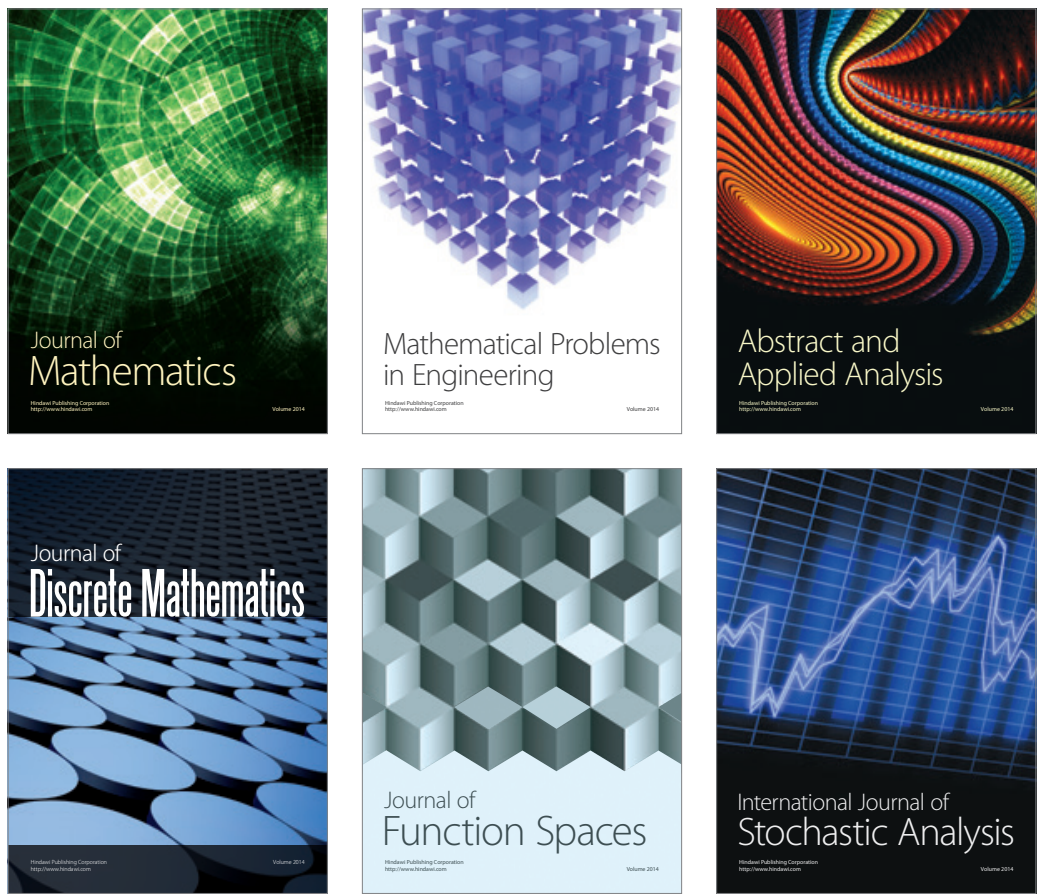

Journal of

Function Spaces

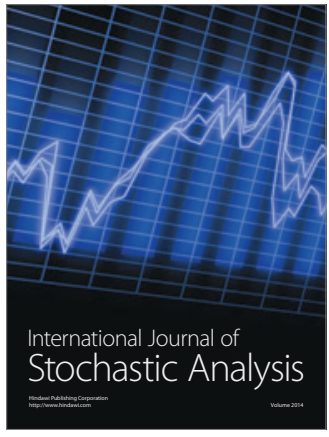

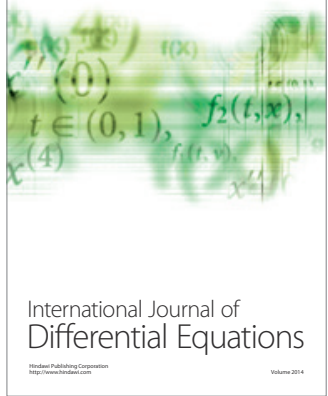
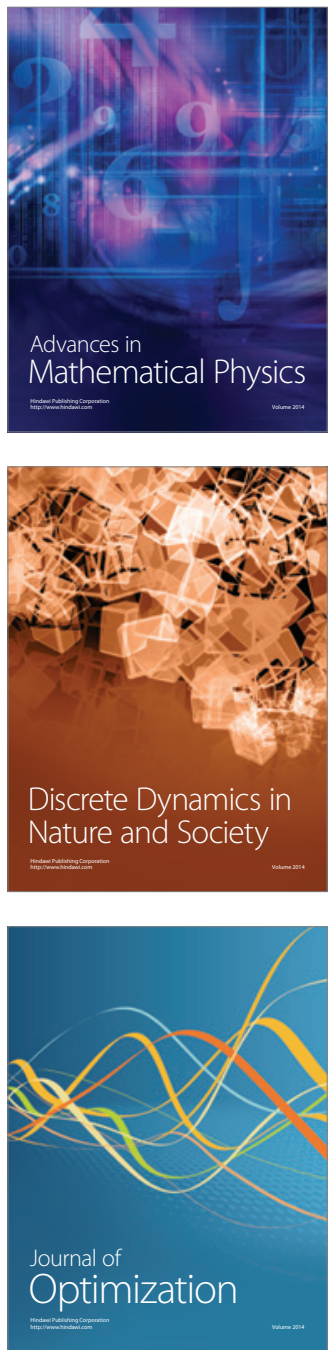